\title{
Q Inversion and Comparison of Influential Factors among Three Methods: CFS, SR, and AA
}

\author{
Yinting $\mathrm{Wu}^{1, *}$ and J. T. $\mathrm{Wu}^{2}$ \\ ${ }^{1}$ School of Geological and Surveying E Mapping Engineering, Chang'an University, \\ $X i^{\prime}$ an 710064, P.R. China. \\ 2 School of CML Engineering $\mathcal{E}$ Architecture, Ningbo Institute of Technology, \\ Zhejiang University, Ningbo 315100, P.R. China.
}

Received 2 November 2018; Accepted 11 January 2019

\begin{abstract}
The goals of this study were to examine factors influencing $Q$ inversion and to provide references for practical application. Three different methods for inverting $Q$ values with VSP data were explored, including centroid frequency shift (CFS), spectral ratio (SR), and amplitude attenuation (AA). Comparison between the CFS and the other two methods was conducted on frequency band widths and low attenuation, wavefield components, interface interference, and thin layers. Results from several sets of VSP modeling data indicated that the CFS method is more stable and accurate for dealing with thin and high $Q$ layers. Frequency band width, especially the presence of high frequencies, influences the inversion effect of all three methods. The wider the band, the better the results. $Q$ inversion from downgoing wavefield was very similar to that of the upgoing wavefield. The CFS method had fewer outliers or skip values from the full wavefield than the other two methods. Moreover, the applications to Q inversion for the set of field VSP data demonstrated that the Q curves from the CFS method coincided with the geological interpretations better than the $Q$ curves of the other methods. Meanwhile, inverse $Q$ filtering shifted the frequency component from $25 \mathrm{~Hz}$ to $35 \mathrm{~Hz}$. The results demonstrated that the Q curve is more sensitive to geological horizons than velocity.
\end{abstract}

AMS subject classifications: 74J25, 86A15, 86A22, 86A60

Key words: $Q$ inversion, centroid frequency shift, amplitude attenuation, spectral ratio, zerooffset VSP data.

\section{Introduction}

Inherent energy attenuation, resulting in high frequency absorption and waveform alteration, reflects the non-elastic property of seismic waves propagating through a medium

${ }^{*}$ Corresponding author. Email addresses: wuyinting0215@163.com (Y. Wu), qingqing@163.com (J. T. Wu) 
[1], and is one of the key factors to seismic prospecting resolution. Experiments have proven that attenuation is sensitive to the porosity, permeability, and fluid properties of the reservoir stratum [2]. Based on the attenuation of different wave types, the quality factor $(Q)$ can be used for the high-precision interpretation of multi-wave and multicomponent data. As a filtering factor, inverse $Q$ filtering can recover the energy loss caused by formation attenuation and enhance the energy of seismic data [3].

A large variety of algorithms have been created and a great amount of research has been done on the process and characteristics of energy attenuation. Changes in amplitude, frequency, phase, and so forth can be used to calculate the inverse $Q$ value in different domains. Rainer [4] contrasted ten methods based on vertical seismic profile (VSP) data. Tommy [3] carried out a comparative study of up to eight attenuation patterns. Specifically, measurements were conducted at the very beginning of the time domain. Since the most noteworthy manifestation of seismic attenuation is the change of amplitude, the first method taken into consideration was the amplitude attenuation method (AA). Ward and Yong [5], Brzostowski and McMechan [6], and Leggett et al. [7] utilized this traditional method in the modification of seismic wave amplitude from observational data when studying attenuation imaging. However, many factors can interfere with the amplitude of seismic waves, including geometric diffusion, scattering, focal types and detector response. It is believed that the estimated values are not reliable.

The spectrum ratio (SR) method is the most commonly used of the techniques involving the frequency domain that are based on changes of the amplitude spectrum. Many seismologists [8-10] have adopted or improved this approach. Dasgupta and Clark [11] used ground seismic data to calculate the $Q$ value. They hypothesized that the SR method would perform very well when it was applied after normal moveout (NMO), and they also found that a linear relationship between frequency and the amount of attenuation could not be established. Chen et al. [12] took advantage of the SR method in the timefrequency domain to prospect a tight gas sandstone reservoir. Zhang et al. [13] calculated spectral ratios of different periods based on the adaptive wavelet technique, with inverted $Q$ values used for inverse $Q$ filtering. This method, however, depends on a number of artificial factors, such as the length and shape of the time window, the slope of the start-stop frequency, and so forth. The SR method is not considered to be very stable and has a high demand for original seismic data $[4,14,15]$.

The centroid frequency shift (CFS) method was proposed by Quan and Harris in 1997 [16]. It is generally regarded to be the realization form of the rise time principle in the frequency domain. Since the centroid frequency of a waveform (or pulse broadening) is not affected by far wavefield geometry diffusion or the transmission/reflection effect, more reliable results can be obtained from centroid frequency shift. Yan et al. [17] used this method on the joint tomography of $Q$ values and velocities in a cross-well; Wang et al. [18] expanded upon this for use one of the main methods for cross-well seismic attenuation imaging. Zhu et al. [19] and $\mathrm{Wu}[20,21]$ applied and analyzed influential factors using zero-offset VSP models. Meanwhile, Zhu et al. [19] noted that, due to the sensitivity of absorption characteristics to frequency, this method would yield high resolution for 
thin layers. When this method was applied to $Q$ estimations using full waveform inversion (FWI) on noisy data, and it was found that the inversion was not seriously affected by moderate amounts of additive noise [22].

This study applies the CFS method and the other two methods to zero-offset VSP model and field data. We analyze and compare the results under the influence of several factors in order to provide a selection basis for calculating attenuation characteristics in practical applications. We only considered intrinsic attenuation; scattering or other causes of seismic data attenuation were not included.

\section{CFS theory and method}

Since the AA method and SR methods for Q inversion are conventional and well-known, their theories are omitted here; references [5] and [14] are recommended for background information. Only the theory and realization of the CFS method are subsequently delineated, under the assumption that the input signal amplitude spectrum is Gaussian [16].

It is assumed that the propagation of seismic waves conforms to linear system theory, so the centroid frequency and variance of the input signal can be defined as:

$$
f_{s}=\frac{\int_{0}^{\infty} f S(f) d f}{\int_{0}^{\infty} S(f) d f}, \quad \sigma_{s}^{2}=\frac{\int_{0}^{\infty}\left(f-f_{s}\right)^{2} S(f) d f}{\int_{0}^{\infty} S(f) d f} .
$$

Similarly, the centroid frequency and variance of the output signal can be defined as:

$$
f_{R}=\frac{\int_{0}^{\infty} f R(f) d f}{\int_{0}^{\infty} R(f) d f}, \quad \sigma_{R}^{2}=\frac{\int_{0}^{\infty}\left(f-f_{R}\right)^{2} R(f) d f}{\int_{0}^{\infty} R(f) d f},
$$

where $f_{S}$ and $\sigma_{S}^{2}$ are the centroid frequency and variance of the input signal $S(f)$, respectively; $f_{R}$ and $\sigma_{R}^{2}$ are the centroid frequency and variance of the output signal $R(f)$, respectively.

When there is intrinsic attenuation in the local layer, the frequency of seismic waves will be changed. The energy attenuation can be calculated by the shift of the main frequency along the path of seismic waves as follows:

$$
\int_{\text {ray }} a_{0} d l=\left(f_{s}-f_{R}\right) / \sigma_{s}^{2}
$$

where $a_{0}$ is the attenuation factor, which is the reciprocal of $Q$; and the integration is carried out through the propagation ray path $d l$.

By solving the right side of Eq. (2.3), the attenuation value on the left side can be determined. However, the source spectrum data $f_{s}$ and $\sigma_{s}^{2} \cdot 10^{3}$ are usually unavailable during the VSP acquisition process. To address this issue, we use adjacent receivers. 
The upper one is regarded as the source, and the lower one as the receiver. In this case, Eq. (2.3) can be written as:

$$
a_{0 i}=\frac{1}{\sigma_{i}^{2}} \frac{\Delta f_{i}}{\Delta z_{i}}, \quad Q_{i}=\frac{\pi f}{a_{0 i} v_{i}}
$$

where $\Delta f_{i}=f_{i+1}-f_{i}$ is the centroid frequency between two receivers; $\Delta z_{i}$ is the distance between two adjacent receivers; $a_{0 i}$ is the attenuation on the section between the receivers; and $v_{i}$ is the velocity of this section. By calculating each of the two adjacent receivers, we can attain the attenuation measurement of the entire wellbore.

To simulate the attenuation in the VSP record and the frequency change, we designed an eight-layer geological model with different $Q$ values - 15, 40, 60, 80, 350, 30, 60, and 100. All of the parameters are listed in Table 1. The forward calculations of the zerooffset VSP record are based on Ganley's theory [23]. Focusing on isotropic and inelastic layered media, this theory adopts the recursive relation to obtain wavefields from the source to any receivers. The calculation is performed in the frequency domain and the model zero-offset VSP record is shown in Fig. 1, where the layer schematic is at top and the corresponding seismic data are at bottom. The layers with different $Q$ values and thicknesses are depicted in different colors.

Table 1: Parameters of the eight-layer geological model.

\begin{tabular}{||ccccc||}
\hline Layer & $\begin{array}{c}\text { Density } \\
\left(\mathrm{kg} / \mathrm{m}^{3}\right)\end{array}$ & $\begin{array}{c}\text { P-wave velocity } \\
(\mathrm{m} / \mathrm{s})\end{array}$ & Q value & $\begin{array}{c}\text { Thickness } \\
(\mathrm{m})\end{array}$ \\
\hline 1st & 1,500 & 800 & 15 & 50 \\
2nd & 1,800 & 1,200 & 40 & 100 \\
3rd & 2,200 & 1,500 & 60 & 100 \\
4th & 2,400 & 2,000 & 80 & 100 \\
5th & 2,800 & 2,450 & 350 & 100 \\
6th & 2,400 & 2,300 & 30 & 100 \\
7th & 2,700 & 3,000 & 60 & 250 \\
8th & 2,800 & 4,500 & 100 & 450 \\
\hline Note: The source is located at a depth of 0 m, and the sixty receivers \\
\multicolumn{5}{|c}{ are located from 25 $\mathrm{m}$ to 1,500 $\mathrm{m}$, at intervals of 25 m. } \\
\hline
\end{tabular}

We can see that the energy of both the downgoing and upgoing wavefields weakens along with transmission distance. It is worth noting that the waveform widens and its frequency decreases significantly with depth, as can be seen in Fig. 2. The solid (green) line represents the source variance and the dotted (blue) line is the centroid frequency. The centroid frequency decreases with distance from $125 \mathrm{~Hz}$ to $35 \mathrm{~Hz}$. And it is very significant that the centroid frequencies of different $Q$ layers exhibit different trends. The lower the $Q$ value, the faster the centroid frequency decrease. The source variance curve also exhibits the same change. Hence, we can see that the attenuation has a great impact 


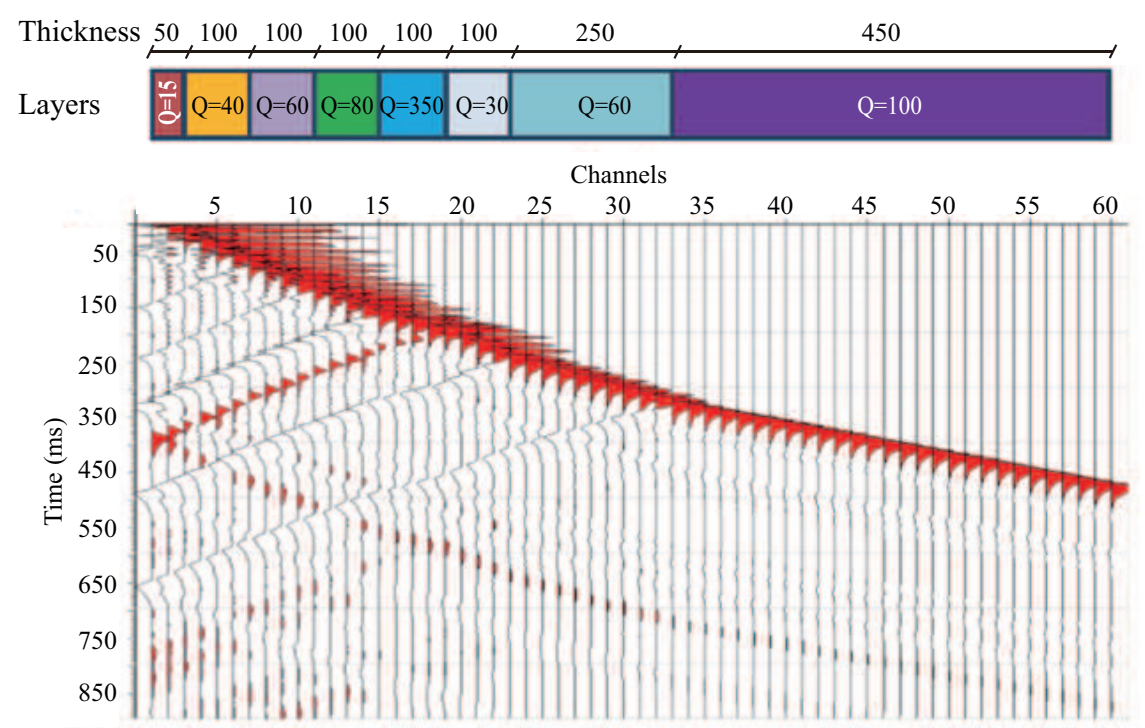

Figure 1: Zero-offset VSP data with attenuation (at bottom) and corresponding geological model (at top).

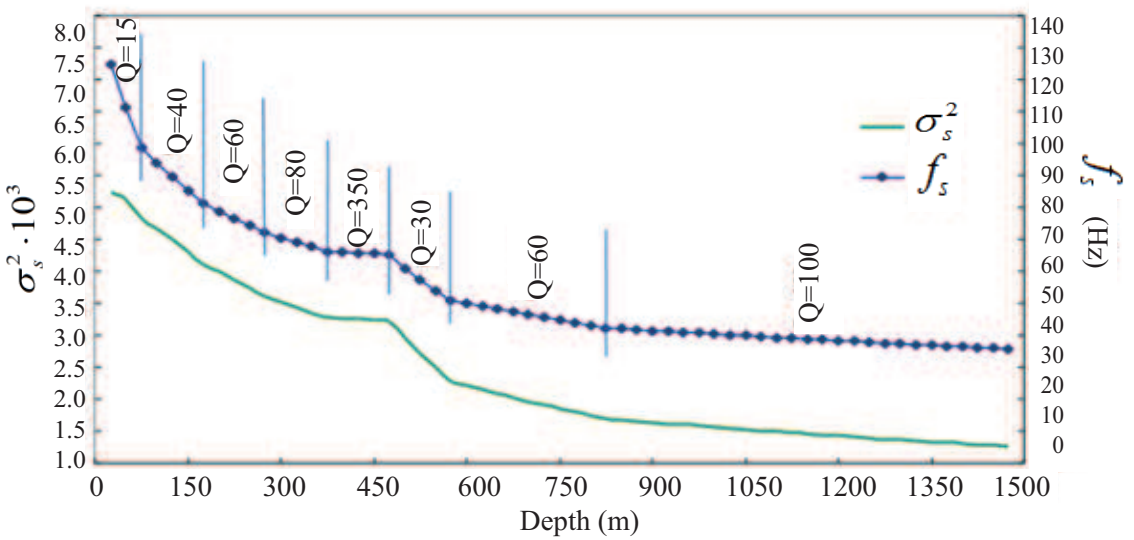

Figure 2: Centroid frequency and variance curves for the simulated VSP data.

on centroid frequency and variance, a fact we can take advantage of when seeking the $Q$ value.

\section{Comparative analysis}

With respect to different influential factors, including frequency range, wavefield component, reflector interference, thin and low attenuation layers, and so on, we designed 
different geological models, calculated and analyzed Q inversion using the CFS and the other two methods SR and AA, and compared their advantages and disadvantages. It should be noted out that the forward modeling was also based on Ganley's attenuation theory [23].

\subsection{Frequency ranges and low attenuation}

To demonstrate the effect of frequency range, especially for layers with low attenuation (high $Q$ value), we utilized the eight-layer geological model mentioned previously in Fig. 1, forwarded the zero-offset VSP data for different frequency band widths, and then inverted each appropriate $Q$ value using the full wavefield.

When the band was smallest $(0-100 \mathrm{~Hz}$, seen in Fig. 3a), the CFS method was somewhat better, and roughly reflected the attenuation trend. According to the CFS, the inversion of the high $Q$ layer was 300 (the theoretical value was 350), with a maximum error rate of $14 \%$. However, the calculation of the SR was $43 \%$, since the inversion value had not yet reached 200. Although it was the best method for characterizing the high $Q$ value boundary, there were too many fluctuations from the SR for the entire well. Meanwhile, the results based on the AA method could not be trusted, since the attenuation of amplitude is not obvious for seismic waves with lower frequencies. Thus, in general, there will be larger errors in the inversion results from methods based on amplitude change, such as the AA method.

However, the inversion results were greatly improved in the wider frequency band $(0-250 \mathrm{~Hz})$ condition and showed the best performance in the band width of $0-400 \mathrm{~Hz}$, as seen in Fig. 3b and Fig. 3c, respectively. With very small fluctuations in the interior of the layers, the three methods were all able to reveal the locations of each interface (seen in Fig. 3c). The inversions for the shallow and base layers were extremely close to their theoretical values. Results from the CFS and SR methods improved significantly. Though the inverted values were lower than the actual measurements, both methods revealed the existence of the high Q layer. The CFS was closer to the value and the SR was closer to the boundary. In addition, the AA method also improved, but its inversion value was just 75, which was lower than the model value.

Comparison of the inversions for the three frequency ranges, $0-100 \mathrm{~Hz}, 0-250 \mathrm{~Hz}$ and $0-400 \mathrm{~Hz}$, led us to conclude that the band width has a great influence on the inversion, especially for high $Q$ strata. From our perspective, the reason for this stems from the fact that a stratum with a high $Q$ value experiences smaller absorption and attenuation effects. Thus, the influence on low frequencies is not obvious, while the opposite effect is true for high frequencies. Therefore, it is difficult to obtain accurate results based on small changes of amplitude or frequency for seismic waves traveling in low attenuation or high Q strata.

When performing the inversion of $Q$ value, it is required that the frequency band of the seismic data be as wide as possible in order to reflect rock attenuation. Its main frequency should also be high enough. With a small frequency range, such as $0-100$ 
(a)

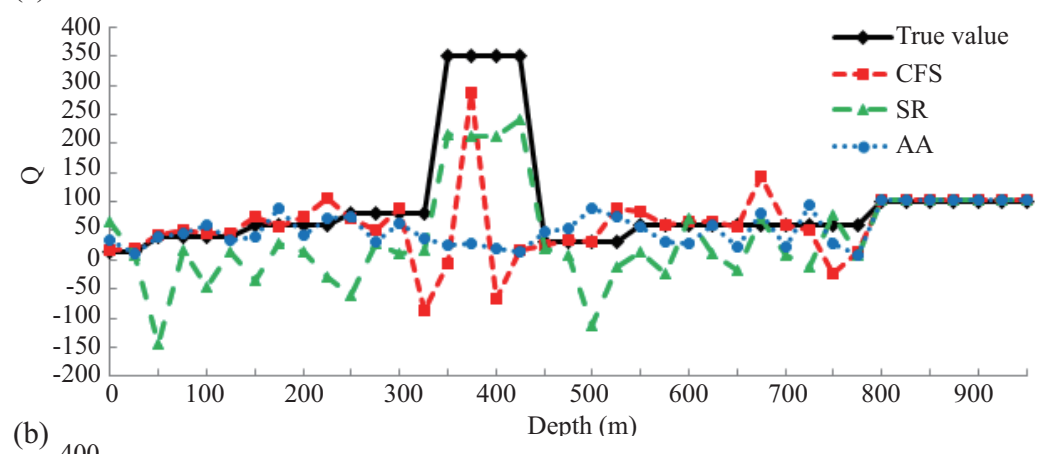

(b)
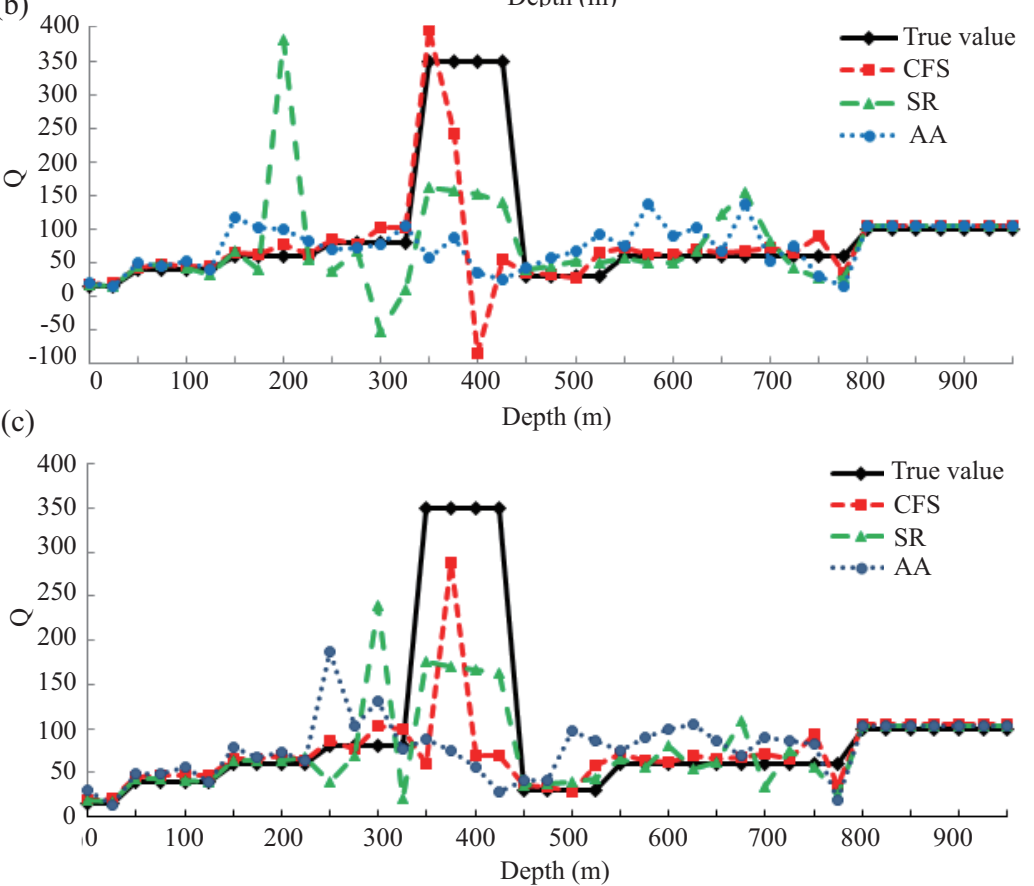

Figure 3: Comparison of inversion Q curves for different frequency band widths: (a) $0-100 \mathrm{~Hz}$; (b) $0-250 \mathrm{~Hz}$; (c) $0-400 \mathrm{~Hz}$.

$\mathrm{Hz}$, a comparison of the CFS and SR methods-both of which are based on frequency change-indicates that the CFS is more stable. Hence, in terms of actual application, we give priority to the CFS method.

\subsection{Wavefield components}

In general, $Q$ value calculation requires only the separated wavefield, such as the downgoing wavefield. Meanwhile, in VSP data processing, a very important step is the separation of the wavefield to acquire the upgoing and downgoing wavefields. In actually, 
it is difficult to completely separate the wavefield since different components may easily mix with one another, especially near the interfaces.

In order to test the effects of the three methods on different wavefield components, a seven-layer geological model was designed. Its parameters are listed in Table 2, and the inversion results are shown in Fig. 4. The frequency range was $0-100 \mathrm{~Hz}$.

Table 2: Parameters of the geological model for different input wavefields test.

\begin{tabular}{||ccccc||}
\hline Layer & $\begin{array}{c}\text { Density } \\
\left(\mathrm{kg} / \mathrm{m}^{3}\right)\end{array}$ & $\begin{array}{c}\text { P-wave velocity } \\
(\mathrm{m} / \mathrm{s})\end{array}$ & Q value & $\begin{array}{c}\text { Thickness } \\
(\mathrm{m})\end{array}$ \\
\hline 1st & 1,500 & 800 & 15 & 50 \\
2nd & 1,800 & 1,200 & 40 & 100 \\
3rd & 2,200 & 1,500 & 60 & 140 \\
4th & 2,400 & 2,000 & 80 & 100 \\
5th & 2,800 & 2,450 & 100 & 300 \\
6th & 2,400 & 2,300 & 40 & 50 \\
7th & 2,700 & 3,000 & 80 & 250 \\
\hline Note: The observation system included 90 receivers at intervals of $10 \mathrm{~m}$. \\
\hline \multicolumn{5}{|l}{}
\end{tabular}

From the results of the upgoing (Fig. 4a) and downgoing (Fig. 4b) wavefields, we can conclude that, disregarding the errors near the interfaces, all three of the methods can reflect $Q$ values with a single component. The upgoing and downgoing wavefields exhibited somewhat similar results. In the case of the mixed wavefield, the inversion was unsatisfactory, with the exception of the first and last layers (Fig. 4c). The reason for this phenomenon is relatively easy to understand. Different wavefields have different propagation directions. Upward and downward waves have opposite energy and frequency attenuation trends. Therefore, when a single wavefield is used, the calculated value should be close to the theoretical value regardless of whether the operation is carried out from top to bottom or from bottom to top. However, if we utilize the mixed wavefield, there will be a large number of computational errors, since it is not consistent with the change of attenuation law.

\subsection{Interface interferences}

The reflection of interfaces interferes with $Q$ inversion. To illustrate the influence of interfaces, the density and velocity of each layer in Table 2 (seen in previous section) are set to the same respective values of $2,000 \mathrm{~kg} / \mathrm{m}^{3}$ and $2,000 \mathrm{~m} / \mathrm{s}$. The inverted $Q$ curves with downgoing wavefields are shown in Fig. 5. In contrast to Fig. 4a, we can see that, in this case the results of all three methods are almost perfect, with the outliers at the interfaces having all but disappeared. All three of the methods accurately show the attenuation characteristics. Therefore, although we think that the interface reflection is an obstruction for the $\mathrm{Q}$ value inversion, it unfortunately could not be avoided. 


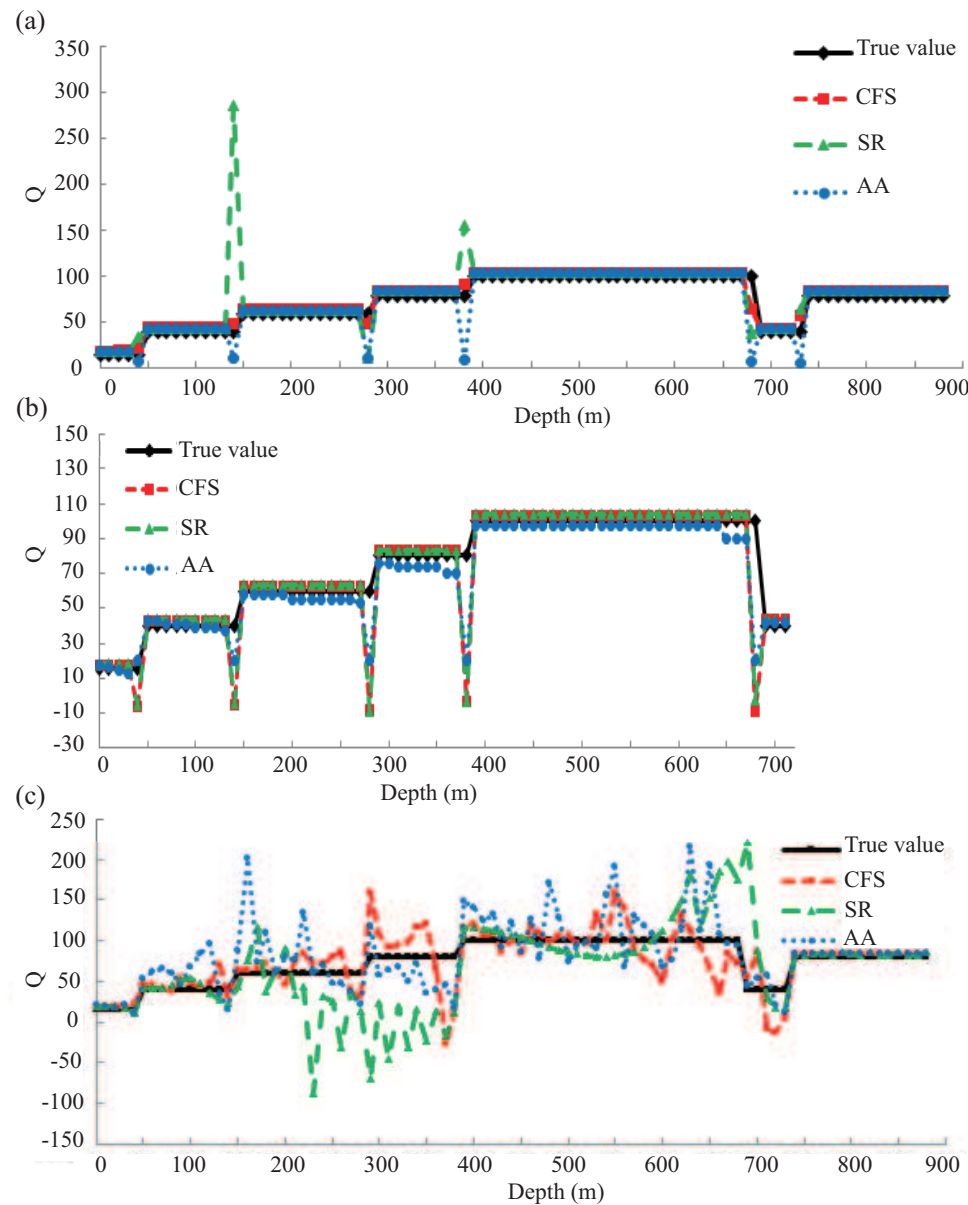

Figure 4: Comparison of inversion $Q$ curves for different wavefield inputs: (a) downgoing wavefield input; (b) upgoing wavefield input; (c) full wavefield (downgoing + upgoing) input.

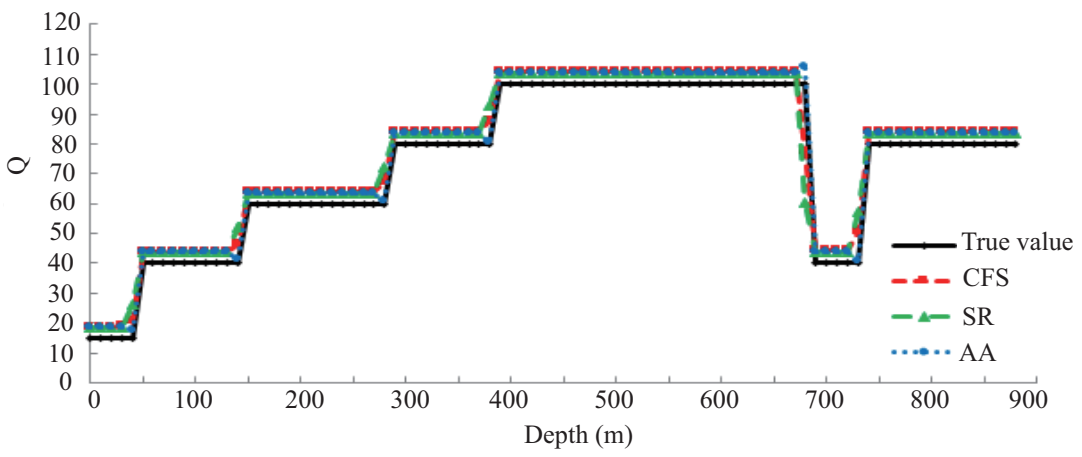

Figure 5: Inversion Q curves for the model in Table 2 with the same density and velocity values of each layer. 


\subsection{Relatively thin layers}

To reveal the reaction ability and precision of the CFS, SR, and AA methods for thin layers, a four-layer model was designed consisting of three relatively thin layers. Their thicknesses were $2 \mathrm{~m}, 6 \mathrm{~m}$ and $3 \mathrm{~m}$, corresponding to $Q$ values of 25,40 , and 50 . The shot point was located at the wellhead, and 90 receivers were located in the borehole from $1 \mathrm{~m}$ to $20 \mathrm{~m}$, at intervals of $1 \mathrm{~m}$. The geological structure and corresponding geometry are shown in Fig. 6a. Q inversion was conducted on the downgoing wavefield, with a frequency range of $0-200 \mathrm{~Hz}$.

By comparing the $Q$ values in Fig. 6b, we can see that all three of the methods reflect the attenuation trend (especially for the last thick layer), and are capable of revealing the absorption characteristics. However, more detailed observations reveal certain differences. The $Q$ values calculated with the CFS method mostly coincided with the theoretical values, with the exception of outliers at $8 \mathrm{~m}$ and $10 \mathrm{~m}$. The error rate was approximately $20 \%$, and the turning points corresponded perfectly to the interfaces and changed smoothly. The $Q$ value for the first layer from the SR method was 10, approximately $60 \%$ beyond the theoretical value of 25 . For the second layer, the inversion appears a large upward trend at $2 \mathrm{~m}$, and fluctuated at $6 \mathrm{~m}$, indicating that the thickness division of the second layer was not accurate enough. The AA method had an outlier at $3 \mathrm{~m}$, which did

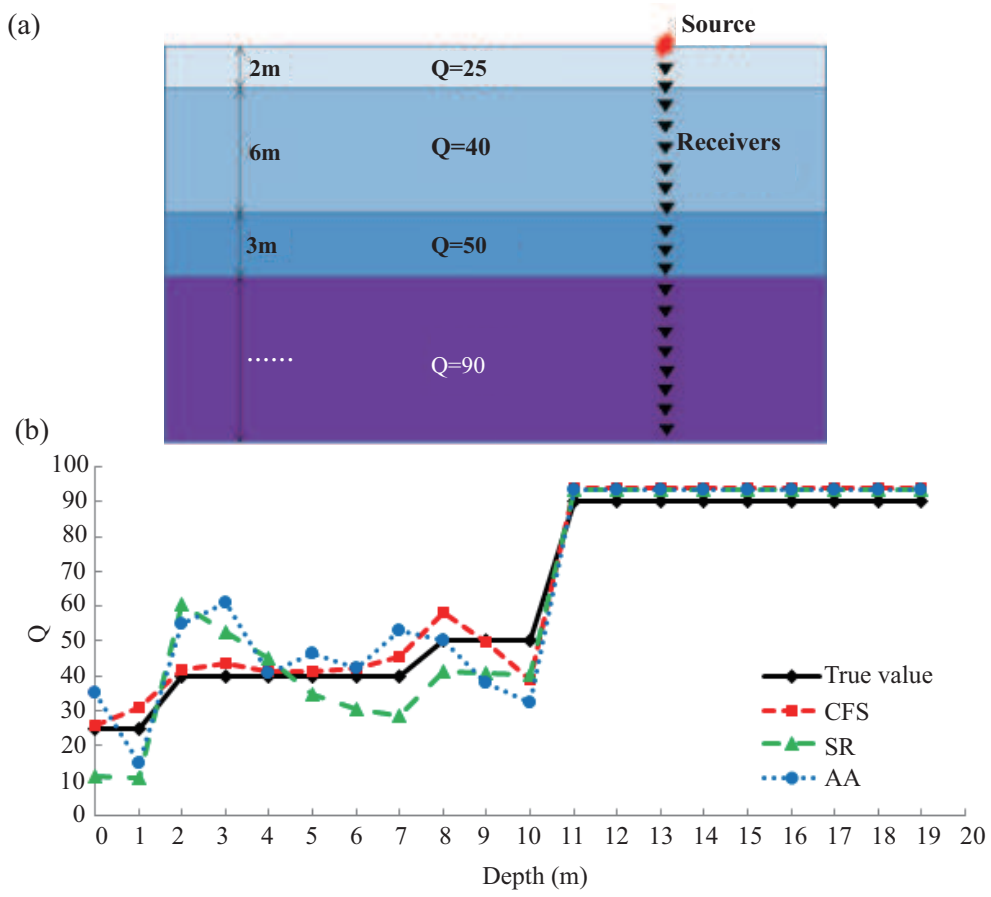

Figure 6: Comparison of the inversion $Q$ curves for the model with three thin layers: (a) the four-layer model with three relatively thin layers and seismic geometry; (b) comparison of inversion $Q$ curves. 
not demonstrate the thickness of the second layer accurately. There was a declination value in the fourth interface of $10 \mathrm{~m}$.

\section{Application with field data}

Based on the above analysis, we believe that the CFS method is more accurate than either the SR or AA under the same conditions for a narrow frequency band, low attenuation, or thin layers. Therefore, the CFS method is recommended for practical application, and a series of field data was processed. We not only used inverse $Q$ values for the paleosol and loess layers in order to attain sensitivity surpassing that of velocity, but also made use of the $Q$ values for inverse $Q$ filtering to compensate for the high frequency component of signals.

\subsection{Q curves for paleosol and loess recognition}

For the zero-offset VSP data from a particular region, the acquisition and observation system parameters were as follows: The source was located near the top of the wellbore at a depth of $0 \mathrm{~m}$; a total of thirty-eight receivers were arranged from 1 to $38 \mathrm{~m}$, at intervals of $1 \mathrm{~m}$, and, since they were shallowly placed they were numbered from largest to smallest. Fig. 7 is the original field record. It is clearly apparent that the downgoing and upgoing waves intersected twice underground, at depths of $20 \mathrm{~m}$ and $30 \mathrm{~m}$, revealing the occurrence of reflection interfaces. The frequency spectrum analyses for the second and seventh channels are shown in Fig. 8. Comparison of the two analyses reveals that the frequency range was reduced from $180 \mathrm{~Hz}$ in the seventh channel to $100 \mathrm{~Hz}$ in the second channel. The absorption of high frequencies was significant. This was in line with the acquisition condition of this region, which includes loose strata near the surface.

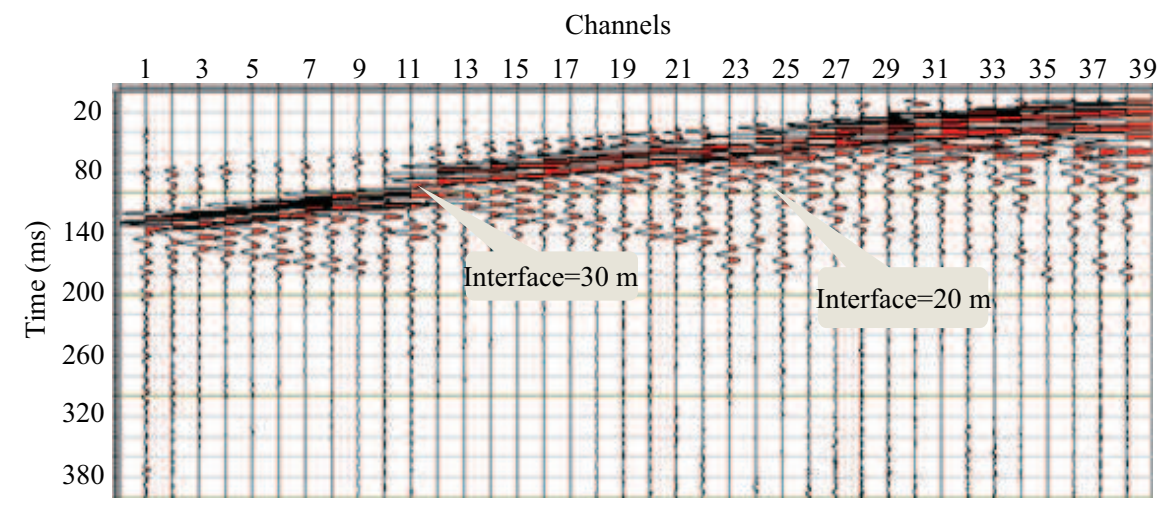

Figure 7: One depiction of the original field data. 
(a)

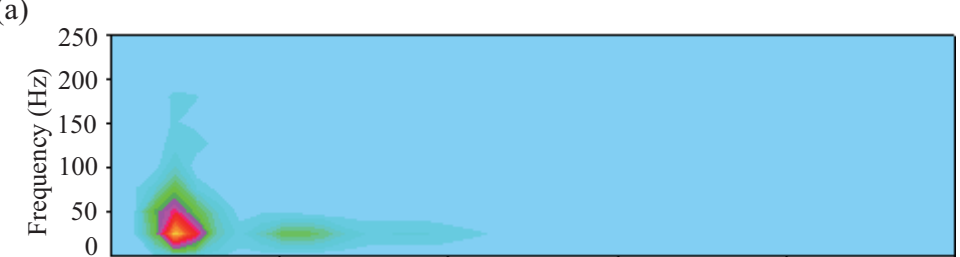

(b)

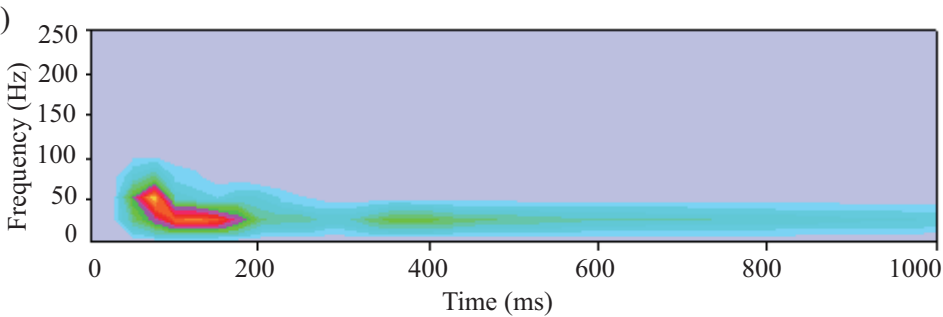

Figure 8: Frequency spectrum analyses of the second (a) and seventh (b) channels.

(a)

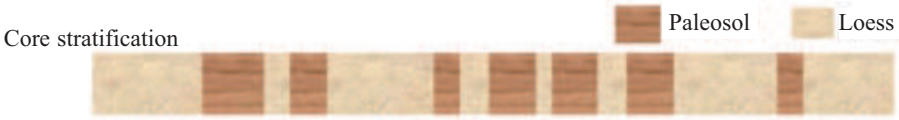

(b)
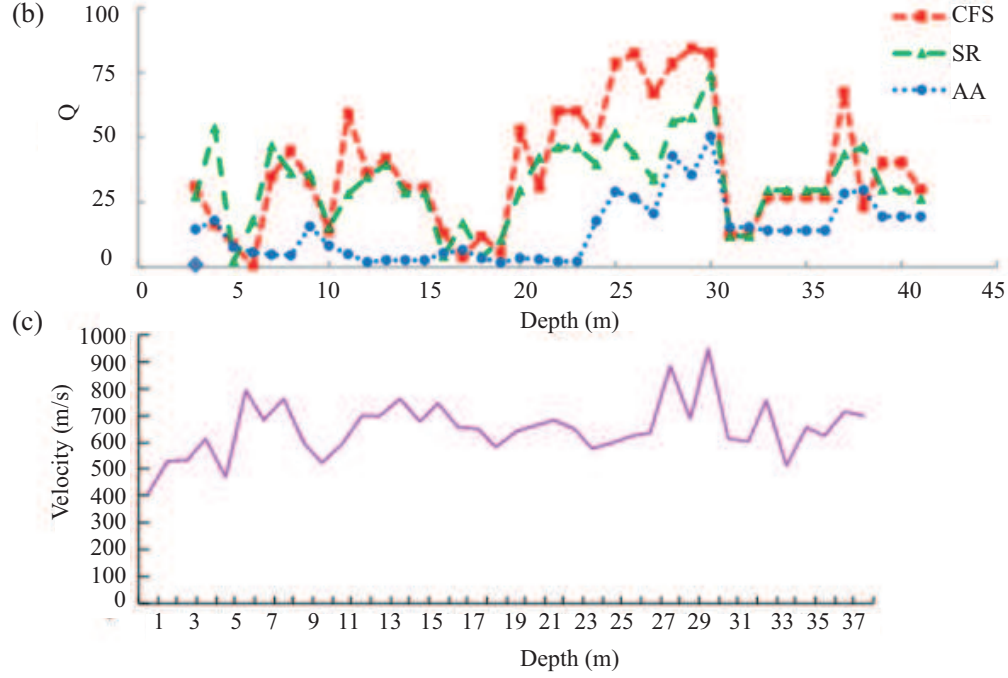

Figure 9: Comparison of the drilling stratifications (a), inversion $Q$ curves (b) and velocity curve (c) for the field data.

The drilling stratifications and corresponding inversion results are shown in Figs. 9a and $9 \mathrm{~b}$, respectively. Relatively speaking, the paleosol (dark color) has strong compaction, compact structure, and hard soil, resulting in less attenuation. The loess (light color) with lower $Q$ values has a strong capability for seismic wave attenuation due to 
its loose and poor compaction. A comparison of the $Q$ curves (Fig. 9b) reveals that the $Q$ values has a strong association with the known reflection interface depth, and were larger in the paleosol formation, while they were smaller values in the loess formation. Thus, the $Q$ values were consistent with the lithological features.

The consistent extent of the CFS method with the strata was the best among the three methods; it accurately reflected the positions of geological horizons. The SR method could capture the main trend of $Q$ value changes. Inversion with the AA method was poor. This is due to the fact that the field data were often disturbed by numerous external factors, and the CFS method was better able to resist disturbance. The $Q$ values from the CFS method were believed to be more sensitive than the velocity curve, as seen in Fig. 9c, since the change of velocity does not reflect the lithology well at each interface, while the $Q$ values are in good agreement with the lithological formations.

\section{2 $Q$ values for inverse $Q$ filtering}

Inverse $\mathrm{Q}$ filtering is a technique used to compensate for the attenuation effect of the earth. This filtering can enhance seismic energy and frequency, especially when compensating for the energy of high frequency seismic waves, since these waves have more attenuation in their high frequency components. However, we have to concede that all of the effects depend primarily on the accuracy of the input $Q$ values. We took advantage of one VSP dataset from an oil field to illustrate $Q$ inversion and filtering.

The acquisition parameters included 220 total channels, a time sample of $500 \mu s, 8,192$ sampling points, and a sampling length of $4,096 \mathrm{~ms}$. The result of $Q$ inversion using the CFS method is displayed in Table 3. This was then utilized as the input for the inverse Q filtering of the adjacent 3D seismic ground data. The records are displayed in Fig. 10. The left and right sides (Figs. 10a and 10b) are the seismic records before and after $\mathrm{Q}$ filtering, respectively. Comparison of the two sides reveals that the continuity of events improved after filtering. In particular, the two demarcated areas were somewhat obscure

Table 3: $Q$ inversion values for the field VSP data (displayed every 10 channels).

\begin{tabular}{||cc|cc|cc||}
\hline $\begin{array}{c}\text { First break time } \\
(\mathrm{ms})\end{array}$ & Q value & $\begin{array}{c}\text { First break time } \\
(\mathrm{ms})\end{array}$ & Q value & $\begin{array}{c}\text { First break time } \\
(\mathrm{ms})\end{array}$ & Q value \\
\hline 130 & 41 & 484 & 71 & 821 & 92 \\
177 & 45 & 525 & 74 & 856 & 93.5 \\
230 & 49 & 567 & 76 & 891 & 95.5 \\
272 & 54 & 611 & 81 & 923 & 96 \\
315 & 57 & 658 & 83 & 956 & 96.5 \\
359 & 62 & 706 & 86 & 989 & 97 \\
402 & 64 & 747 & 88 & 1,021 & 97.5 \\
443 & 68 & 783 & 90 & & \\
\hline
\end{tabular}




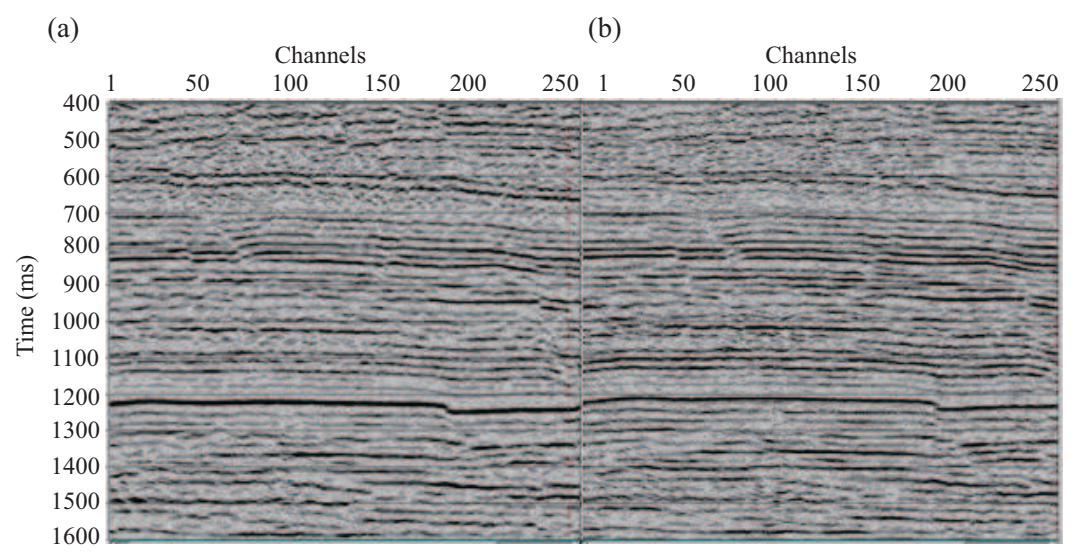

Figure 10: Comparison of the seismic record before (a) and after (b) inverse $Q$ filtering.

before processing, but became distinguishable and continuous, with enhanced energy following inverse filtering.

Fig. 11 shows the effect of inverse $Q$ filtering. Focusing on amplitudes exceeding 0.5, the frequency range mainly fell between $12-44 \mathrm{~Hz}$ in the original record, while shifting to $16-50 \mathrm{~Hz}$ after processing. Moreover, the dominant frequency changed from $25 \mathrm{~Hz}$ to $35 \mathrm{~Hz}$.

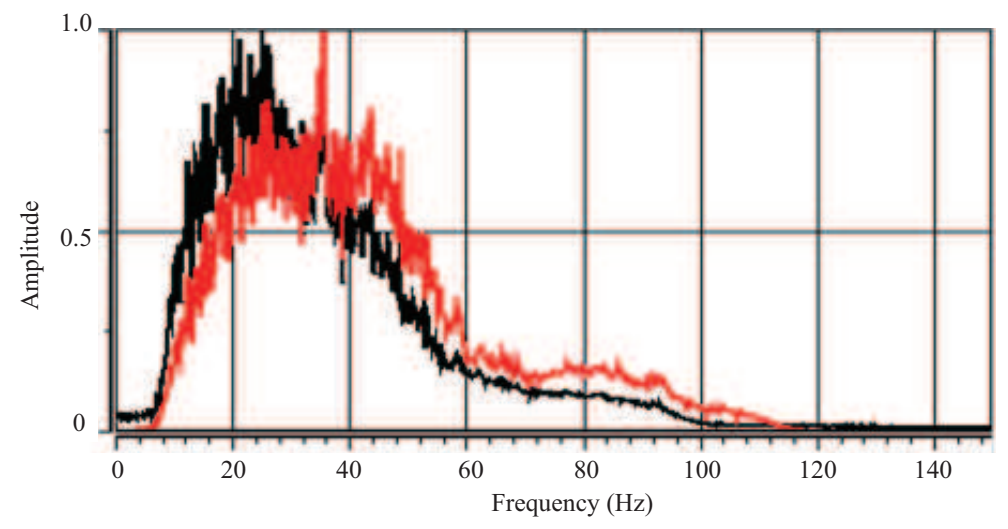

Figure 11: Comparison of the spectrum before (black line) and after (red line) inverse $Q$ filtering.

\section{Conclusions}

Comparing among the results from the CFS, AA, and SR methods, it was discovered that the CFS method is more effective for revealing different layers and is also more stable for a mixed wavefield. For thin layers, the results from the CFS method yielded fewer 
errors. The AA and SR methods had error rates of more than $60 \%$. For the high Q layers, the largest error rate of the CFS method was $14 \%$, while those of the other two methods ranged as high as $43 \%$. Thus, we think that results from the CFS method are more plausible than those from either the SR or the AA method for thin and weakly attenuating media. In addition, based on the inversions of different frequency ranges, we can conclude that the wider the frequency range, the better the inversion, especially for the CFS and SR methods. Moreover, the application of a series of VSP field data revealed that the $\mathrm{Q}$ curve of the CFS method coincided with the geological layers better than the Q curves of the other two methods, which barely reflected the main trend. In summary, although the CFS method is relatively new, it is generally more robust and can be considered a promising technique.

In subsequent research, we will attempt to combine the CFS method with timefrequency analysis, and to explore new approaches which will be preferable for field seismic data and non-stationary signals.

\section{Acknowledgments}

This work is supported by the Fundamental Research Funds for the Central Universities (Grant No. 300102268212), Postdoctoral Science Foundation (2013M540756, 2014T70925) and the Shaanxi Natural Science Foundation (2014JQ2-4019). The authors wish to acknowledge the support and contributions of the project sponsors, other participating institutions and their personnel.

\section{References}

[1] T. W. Spencer, Seismic wave attenuation in nonresolvable cyclic stratification, Geophys., 42(5) (1997), 939-949.

[2] K. F. Xin, Z. C. Li, Y. G. Wang, S. J. Chu, Z. T. Ma, Formation equivalent absorption coefficient inversion, Geophys Prospect Petrol, 40(4) (2001), 14-20.

[3] T. Tommy, U. Bjorn, Comparison of seismic attenuation models using zero-offset seismic profiling (VSP) data, Geophys., 70(2) (2005).

[4] T. Rainer, The determination of the seismic quality factor Q from VSP data. A comparison of different computational methods, Geophys. Prospect., 39 (1991), 1-27.

[5] R.W. Ward, C. Y. Young, Mapping seismic attenuation within geothermal systems using teleseisms with application to the Geysers-Clear Lake region, J. Geophys. Res-sol. Ea., 85 (1980), 5227-5236.

[6] M. A. Brzostowski, G. McMechan, 3-D tomographic imaging of near-surface seismic velocity and attenuation, Geophys., 57 (1992), 396-403.

[7] M. Leggett, N. R. Goulty, J. E. Kragh, Study of travel time and amplitude time-lapse tomography using physical model data, Geophys. Prospect., 4 (1993), 599-619.

[8] R.W. Ward, M. N. Toksoz, Causes of regional variation of magnitudes, B. Seismol. Soc. AM., 61 (1971), 649-670.

[9] R. K. Elsley, B. R. Tittmann, H. R. Nadler, L. A. Ahlberg, Defect characterization by ultrasonic signal process techniques, Proc. Ultrason. Symp., IEEE, (1977), 48-52. 
[10] F. M. Sears, B. P. Bonner, Ultrasonic attenuation measurement by spectral ratios utilizing signal processing techniques, IEEE Trans. Geosci. Remote. Sens., 19(2) (1981), 95-99.

[11] R. Dasgupta, R. A. Clark, Estimation of Q from surface seismic reflection data, Geophys., 63 (1998), 2120-2128.

[12] W. S. Chen, L. P. Guan, Z. C. Li, K. Xu, Prestack Q-inversion based on generalized S transform, Geophys. Prospect Petrol., 53 (2014), 706-712.

[13] F. C. Zhang, X. X. Zhang, L. Q. Zhang, Extraction method for quality factor Q based on adaptive wavelet decomposition, Geophys., 55 (2016), 41-48.

[14] M. Sams, D. Goldberg, The validity of $Q$ estimates from bore-hole data using spectral ratios, Geophys., 55 (1990), 97-101.

[15] R. Tonn, The determination of the seismic quality factor Q from VSP data: A comparison of different computational methods, Geophys. Prospect., 39 (1991), 1-27.

[16] Y. L. Quan, J. M. Harris, Seismic attenuation tomography using the frequency shift method, Geophys., 62 (1997), 895-905.

[17] Y. S. Yan, M. L. Yi, X. Wei, W. M. Wan, Joint tomographic imaging for cross-hole seismic velocity and Q value, Oil Geophys. Prospect., 36 (2001), 9-17.

[18] H. Wang, X. Chang, F. Gao, Several methods of crosswell seismic attenuation tomography, Prog. Geophys., 16 (2001), 104-109.

[19] D. Zhu, X. G. Min, H. M. Gu, Inversion of attention coefficient based on frequency shift, Prog. Explor. Geophys., 29 (2006), 30-33.

[20] Y. T. Wu, The forward and inverse of $Q$ value using zero-offset VSP data, Xi'an: Chang'an University, 2008.

[21] Y. T. Wu, G. M. Zhu, CFS method for inversion of Q value from zero-offset VSP data, Prog. Geophys., 25 (2010), 1897-1904.

[22] R. L. Laurence, V. Fereidoon, R. P. Bording, Short note: seismic-Q estimation using full waveform inversion of noisy data-a feasibility study, Can. J. explor. geophys., 38(1) (2013), 15-20.

[23] D. C. Ganley, A method for calculating synthetic seismograms which include the effects of absorption and dispersion, Geophys., 46(8) (1981), 1100-1107. 